\title{
Correlation between Macroscopic Elasticity and Chain Dynamics of Natural Rubber during Vulcanization as Determined by a Unique Rheo- NMR Combination
}

\author{
Shouliang Nie, ${ }^{\text {a }}$ Karl-Friedrich Ratzsch, ${ }^{\mathrm{b}}$ Stephan L. Grage, ${ }^{\mathrm{c}}$ Jonas Keller, ${ }^{\mathrm{a}}$ \\ Anne S. Ulrich, ${ }^{\mathrm{c}}$ Jorge Lacayo-Pineda, ${ }^{\mathrm{d}}$ and Manfred Wilhelm ${ }^{\mathrm{a}}{ }^{*}$ \\ ${ }^{\text {a }}$ Institute for Technical Chemistry and Polymer Chemistry, Karlsruhe Institute of Technology (KIT), \\ 76131 Karlsruhe, Germany; \\ ${ }^{\mathrm{b}}$ Bruker BioSpin GmbH, 76287 Rheinstetten, Germany; \\ ${ }^{\mathrm{c}}$ Institute of Biological Interfaces (IBG-2) and Institute of Organic Chemistry (IOC), Karlsruhe Institute \\ of Technology (KIT), 76131 Karlsruhe, Germany; \\ ${ }^{\mathrm{d}}$ Research and Development, Continental Reifen Deutschland GmbH, 30419 Hannover, Germany.
}

\section{Comparison of $x \times 4$ and Hahn echo experiments:}

To justify whether the transverse relaxation signal from the $\mathrm{xx} 4$ sequence is as same as the intensity acquired from Hahn echo approach, both $\mathrm{xx} 4$ and Hahn echo experiments were performed on the same material to compare. The experiments were conducted on a mq20 NMR Analyzer (Bruker, Rheinstetten, Germany) with a ${ }^{1} \mathrm{H}$ Larmor frequency of $\omega_{L} / 2 \pi=19.95 \mathrm{MHz}$ and dead-time of $11 \mu \mathrm{s}$. The $90^{\circ}$ and $180^{\circ}$ pulse lengths are $2.9 \mu \mathrm{s}$ and $=5.0 \mu \mathrm{s}$, respectively. Compared to the rheo-NMR setup, mq20 possesses more homogeneous $B_{0}$ static magnetic filed and stable temperature control thus provides higher accuracy of the results. Further more, mq20 is more convenient to conduct the experiment while the setup of rheo-NMR is more sophisticated. The examined sample is the fully crosslinked EV (i. e., EV100). The rubber vulcanizate was cut into small granules and filled into a O.D. $=10 \mathrm{~mm}$ diameter NMR glass tube with a pile height below $8 \mathrm{~mm}$. All of the experiments were conducted at $120{ }^{\circ} \mathrm{C}\left(20{ }^{\circ} \mathrm{C}\right.$ lower than the vulcanization measurements to avoid/suppress the influence of further crosslinking during the experiments) with a socking time of $15 \mathrm{~min}$ before the measurement. The setting parameters for $\mathrm{xx} 4$ here were the same as the vulcanization test on rheo-NMR. Regarding the individual Hahn echo experiments, the echo time was set as 1.05, 2.10, 3.15, 
$4.20,5.20,6.30,8.40,10.50,12.60$, respectively, which will cover the typical relaxation signal range of $\mathrm{xx} 4$ part. The scan number was 32 .

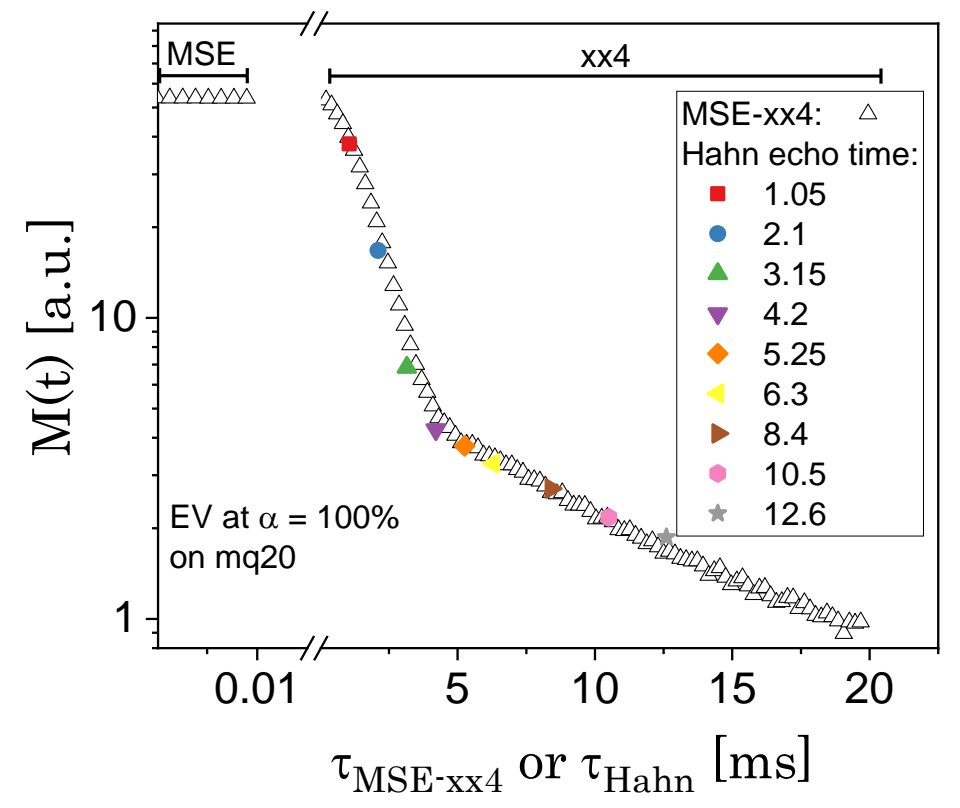

Figure S1. The NMR transverse relaxation signal of a fully crosslinked EV sample acquired on a mq20 NMR analyzer from single MSE-xx4 experiment (open symbol) and multiple Hahn echo experiments with different echo time (solid symbols).

The magnetization decay received from the single MSE-xx4 experiment is compared with the echo peak intensities from multiple Hahn echo experiments as shown in Figure S1. Notice here the FID decay in MSE acquired from mq20 is not as fast as that acquired from the rheo-NMR setup (see Figure $4 \mathrm{~b}$ ). This is due to the more homogeneous $B_{0}$ filed of mq20. Furthermore, the transverse relaxation in $\mathrm{xx} 4$ part acquired from mq20 is less noisy. It is found that the signal from $\mathrm{xx} 4$ part overlaps well to the Hahn echo peak intensities at the same echo train time. Therefore, the transverse relaxation curves from a single $\mathrm{xx} 4$ experiment can be treated equivalently as the results from the Hahn echo approach. While the latter requires multiple experiments for one transverse decay curve thus is not time-efficient. Here, compared to the original CPMG sequence, the $\mathrm{xx} 4$ pulse sequence was using an alternating-phase sequence (see section 2.2.2) to avoid spin-locking effects on the $T_{2}$ measurements. ${ }^{1,2}$ In CPMG experiments without phase alternating, the $T_{2}$ time constants decrease with the prolonging of inter-pulse spacing. While it was also suggested that the dependency of $T_{2}$ time on the inter-pulse spacing in CPMG is not due to spin-locking, where the diffusional motion of polymer melts during $T_{2}$ measurements should be taken into account. ${ }^{3}$ 


\section{NMR data treatment:}

Eq 3 was examined on the fully crosslinked CV or EV samples (from three repeating tests of each sample) and the fitting results are listed in Table S1. In the fully crosslinked sample, the major component $\left(I_{S}=77-89 \%\right)$ response to the short relaxation time, $T_{2 s}$, around $1.5 \mathrm{~ms}$. The compression factor $\beta$ is experimentally determined to be around 1.6 while the transverse relaxation time of $T_{2 l}$ is approximately $5 \mathrm{~ms}$.

Table S1. Fitting results of eq 3 on CV and EV samples (three specimens of each) at fully crosslinked state (at $\alpha=100 \%$ ).

\begin{tabular}{lrrrrrr}
\hline Samples & $\boldsymbol{I}_{\boldsymbol{s}}$ & $\boldsymbol{T}_{2 \boldsymbol{s}}$ & $\boldsymbol{\beta}$ & $\boldsymbol{I}_{\boldsymbol{l}}$ & $\boldsymbol{T}_{\mathbf{2 l}}$ & $\boldsymbol{R}^{\mathbf{2}}$ \\
\cline { 2 - 7 } fully crosslinked: & - & $\mathrm{ms}$ & - & - & $\mathrm{ms}$ & - \\
\hline CV100\%\#1 & 0.89 & 1.48 & 1.58 & 0.11 & 5.54 & 0.9984 \\
CV100\%\#2 & 0.87 & 1.43 & 1.54 & 0.13 & 4.77 & 0.9963 \\
CV100\%\#3 & 0.89 & 1.50 & 1.56 & 0.11 & 4.76 & 0.9982 \\
EV100\%\#1 & 0.77 & 1.59 & 1.62 & 0.23 & 4.32 & 0.9982 \\
EV100\%\#2 & 0.80 & 1.57 & 1.58 & 0.20 & 4.33 & 0.9984 \\
EV100\%\#3 & 0.78 & 1.60 & 1.62 & 0.22 & 4.28 & 0.9985 \\
\hline average & 0.83 & 1.53 & $\mathbf{1 . 5 8}$ & 0.17 & $\mathbf{4 . 6 7}$ & 0.9980 \\
\hline
\end{tabular}

To reduce the number of fitting parameters and increase the fitting stability, eq 3 was fitting on the NMR data along vulcanization with fixed $\beta(=1.6)$ and $T_{2 l}(=5 \mathrm{~ms})$. One exemplar result is shown in Figure $S 2$ with the evolution of the three free fitting parameters, $I_{s}, I_{l}$ and $1 / T_{2 s}$, along the vulcanization of CV sample. The level of $I_{s}$ and $I_{l}$ before $500 \mathrm{~s}$ were not stable and $I_{l}$ was even close to zero. This might result from fitting artifacts, temperature adjustments and additionally from the difficulty to distinguish the two fractions with short or long apparent $T_{2}$ times. In addition, it should be stressed here that the $I_{s}, I_{l}$ are not necessary the fraction of the inter-crosslink chains and free dangling chain ends since eq 3 is only a phenomenological description of the relaxation decay. In crosslinking induction period, the $T_{2 s}$ value obtained from fitting was close to $5 \mathrm{~ms}$, which was the same level of the fixed $T_{2 l}$. It means that a single Weibull term in eq 3 could describe the starting material. After $500 \mathrm{~s}, I_{s}$, started to increase, consequently reducing its counterpart, $I_{l}$. However, the $1 / T_{2 s}$ was changing smoothly along the whole vulcanization course. Among the three NMR parameters in eq 3 , it is found that $1 / T_{2 s}$ 
exhibited the most drastic change upon crosslinking, i.e. it increased almost 3 times, whereas $I_{S}$ and $I_{l}$ only changed by about $20 \%$.

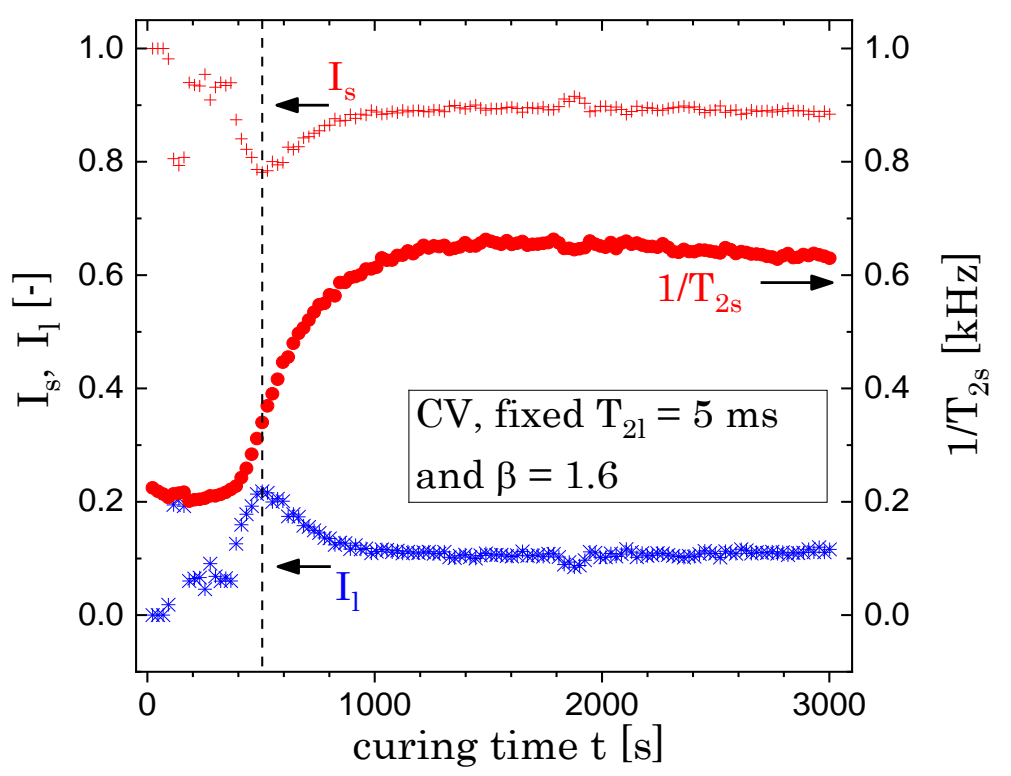

Figure S2. Time evolution of the NMR parameters, $I_{s}, I_{l}$ and $1 / T_{2 s}$ from eq 3 with fixed values of $\beta=1.6$ and $T_{2 l}=5 \mathrm{~ms}$, during the vulcanization of a $\mathrm{CV}$ sample. The term $1 / T_{2 s}$ exhibits the most drastic change. The dashed line indicates the unstable change of $I_{S}$ and $I_{l}$ at the beginning.

\section{References:}

1. Suh, B. J.; Borsa, F.; Torgeson, D. R. (1994). Use of an alternating-phase CPMG sequence to avoid spin-locking effects in T2 measurements in solids. J. Magn. Reson., Ser. A 1994, 110, 58-61.

2. Höpfner, J.; Guthausen, G.; Saalwächter, K.; Wilhelm, M. Network structure and inhomogeneities of model and commercial polyelectrolyte hydrogels as investigated by low-field proton NMR techniques. Macromolecules 2014, 47, 4251-4265.

3. Whittaker, A. K.; Bremner, T.; Zelaya, F. O. The effect of field inhomogeneities and molecular diffusion on the NMR transverse relaxation behaviour of polymer melts. Polymer 1995, 36, 2159-2164. 